\title{
Clinical profile, in-hospital outcome and associated factors of stroke after the start of a standard organized stroke care unit at university of Gondar hospital, northwest Ethiopia.
}

\author{
Seid Getahun Abdella \\ Wollo University \\ Nebiyu Bekele Gebi \\ University of Gondar \\ Ermias Shenkutie Gerffie \\ University of Gondar \\ Koku Sisay Tamirat ( $\sim$ kokusisay23@gmail.com ) \\ University of Gondar
}

Research article

Keywords: In hospital outcome, Mortality, poor outcome, stroke care unit, Gondar

Posted Date: May 13th, 2019

DOI: https://doi.org/10.21203/rs.2.431/v2

License: (c) (i) This work is licensed under a Creative Commons Attribution 4.0 International License.

Read Full License 


\section{Abstract}

Background: Epidemiological transitions and widespread risk factors made stroke common health problem in sub-Saharan countries in the early age. Stroke management largely depends on non-drug interventions. Stroke care units are facilities in hospitals which increased patient survival, return home, and regain independence in daily activities. This study was aimed to assess clinical profile, in-hospital outcome and its associated factors of stroke after the start of a standard organized stroke care unit in the study area. Method: An institution based cross-sectional study was employed from July 2015 to September 2017. A total of 151 stroke patients with computed tomography (CT) scan result were included in the study. Data were collected using structured questionnaire from secondary sources of patient medical records. In-hospital mortality and poor post-stroke disability (greater functional impairment, when Modified Rankin Scale score $(\mathrm{mRS}) \geq 3$ ) were outcome variables. Binary logistic regression model was fitted to identify predictor variables. Adjusted Odds Ratio (OR) with a 95\% confidence interval $(\mathrm{Cl})$ used to assess the strength of association. Variables with $\mathrm{p}$-value less than 0.05 in the multi-variable regression model was considered as significantly associated with the dependent variables. Result: Ischemic stroke (60.3\%) subtype was the most common. The median age at presentation was 65 (IQR: $55-75)$ years. Hypertension (49.7\%) and carotid atherosclerosis (54.7\%) were the most commonly identified risk factors. Overall in-hospital mortality was 9.3\% (95\% Cl: 5.2\%-15.1\%), poor post-stroke disability was $55.6 \%(95 \% \mathrm{Cl}: 47.3 \%-63.7 \%)$, and median length of hospital stay was 10 (IQR: 7-14) days. Being male (AOR=0.19, 95\% Cl: 0.0380 .97 ), longer in-hospital stays (AOR=0.21, 95\% Cl: 0.0480 .93 ) were significant predictors of in-hospital mortality. Furthermore increased ICP (AOR=2.81, $95 \% \mathrm{Cl}$ : 1.226 .92$)$ was also the predictor of poor post-stroke disability at discharge. Conclusion: Ischemic stroke was the most common stroke subtype. In-hospital mortality was relatively lower. However, greater functional impairment was high at discharge. Stroke morality was observed lower among male and longer in-hospital stay patients, while evidence of increased intracranial pressure was associated with poor post-stroke disability at discharge.

\section{Background}

The epidemics of non-communicable diseases result a devastating health consequences [1]. Cardiovascular diseases are the leading causes of disability and death globally [1-3]. Stroke is caused by, the interruption of blood supply to the brain due from blood vessel burst or blockage which is characterized by neurological deficits[4]. Worldwide Stroke is the second leading cause of death and the third leading cause of disability[2]. Globally, majority of stroke related mortality and disabilities occur in low- and middle-income countries $[1,2,5]$.

Significant reductions of stroke incidence observed in developed countries. However, in the last four decades stroke incidence has doubled in developing countries[3, 6]. Ischemic stroke is the most prevalent and hemorrhagic stroke is the most fatal stroke subtypes[7]. African countries faced the double burden from infectious and NCD like stroke. Epidemiological transitions and widespread of risk factors made stroke the common health problem in sub-Saharan countries in the early age[2]. 
Stroke management largely relied on treatment of complications and supportive care in health facilities. Stroke managed in different health facilities like hospital general wards, intensive care units, and stroke care units. Stroke care units show proven effectiveness in improving stroke outcome like increasing patient's survival, return home, and regain independence in daily activities [8-11]. Evidences from previous studies, stroke management in stroke care units decreased mortality by $25 \%$ compared to general hospitals[12, 13].

Organized stroke care units are facilities, which provide stroke care services by a team of health care workers, such as nurses, doctors, and therapists who specialize in monitoring stroke patients. Since July 2013 Gondar University hospital with the collaboration of Southampton University established organized stroke care unit. Trained nurses and neurologists regularly follow the progress of patients. Stroke care unit was new not only in the hospital but also in the country. No study was conducted after the start of organized stroke care unit in the study area.

Therefore, this study was aimed to assess clinical profile, in-hospital outcomes and associated factors of stroke after the start of standard organized stroke care unit at the university of Gondar hospital. This research could provide an insight about the impacts of stroke care unit at hospital level and be used to inform respective stakeholders for strengthening and establishment isolated stroke care unit in a university of Gondar and other hospitals.

\section{Methods}

\section{Study setting}

The study was conducted at the University of Gondar Hospital, which is the only comprehensive, specialized hospital in Gondar City, the Amhara National Regional State, Ethiopia. More than 450, 000 peoples visited the hospital every year for different health care services. The hospital also serves as a referral center for a population of more than five million people in the surrounding catchment area which has varying climatic and geographical distributions. A standard stroke care unit was established at the university of Gondar comprehensives specialized hospital since 2013 with the collaboration of Southampton University for technical and material support.

The stroke care unit has four beds and dedicated staffs such as neurologist, physiotherapists, and nurses who provide stroke care regularly. In addition to human resource the unit equipped with essential materials for stroke care unit. Before the establishment of the unit all patients who diagnosed to have stroke were admitted in general medical wards with other patients like HIV and DM and there were no dedicated and trained staff for stroke patients. The stroke care unit had treatment protocol to support patients.

\section{Population and sample}


An institution based cross-sectional design was employed from July 12015 to September 20 2017. All stroke patients who were admitted and had treatment follow up at the University of Gondar hospital, internal medicine ward stroke care compartment were selected as study population. All patients with confirmed diagnosis of stroke of new onset and admitted to stroke care unit within seven days of diagnosis were included in the study. In fact there were no patients presented delayed than seven day in the study. Diagnosis of stroke is initially clinical and then confirmed by CT scan. The WHO case definition for stroke is patients with clinical manifestations and should be confirmed with CT scan imagining. Despite the cost of CT scan many patients with stroke do manage to get it. The sample size determine using the assumptions of rule of thumb because of absence of important parameters and rarity of the case. All stroke patients who were admitted and got service in the unit during the study were included. A total of 156 stroke patient medical records were identified during the study period. Of which five charts were excluded due to incomplete data for major variables, like in- hospital outcome and clinical variables. Finally 151 patients were the final sample size for the study.

\section{Data collection and variables}

Data were collected by internal medicine residents using a checklist sheet from the patient medical records. Two medical residents were recruited as data collectors and one medical resident as supervisor, a one day training was given to them on how to extract data from medical records. Checklist completeness and consistency checked every day by supervisor and principal investigator.

In-hospital mortality and poor post-stroke disability (greater functional impairment, when Modified Rankin Scale score $\geq 3$ ) were outcome variables. The checklist contained socio-demographic data, HIV status, and type of stroke, common risk factors (hypertension, diabetes mellitus, substance use, neurologic risk factors and atrial fibrillation) and possible treatment outcomes of patients. Poor post-stroke disability (greater functional impairment): when a patient had Modified Rankin Score (mRS) greater than or equals to 3 at discharge[14]. Longer in-hospital stay: patients who stay admitted in hospitals for more than 10 days.

\section{Data management and analysis}

Collected data were checked for consistency, cleaned, coded and entered to Epi Info version 7, and exported to SPSS version 22 for further analysis. Descriptive measures summarized by using frequency tables and graphs. Binary logistic regression was fitted to identify predictor variables of in-hospital mortality and post-discharge poor disability. An Adjusted Odds Ratio (OR) with a 95\% confidence interval was used to assess the strength of association. Variables with p-value less than 0.05 in the multi-variable considered as significant predictors of mortality and poor post-discharge disability.

\section{Results}




\section{Socio demographic characteristics}

Out of total participants, half of them (50.3\%) were females and most patients $(63.7 \%)$ were from rural areas. The median age at the presentation was 65 (IQR: 55-75) years old. Stroke in the young age group(less than 40 years) accounted for $6.6 \%$ of cases (Table 1 ).

\section{Risk factors and co-morbidities}

Antecedent hypertension (50.3\%) found to be the most common risk factor and nearly two third (65.3\%) of patients were on regular medication. History of antecedent hypertension was identified among $44.7 \%$ and $55.3 \%$ of patients with hemorrhagic and ischemic stroke respectively. Diabetes mellitus (DM) was identified as a risk factor among (8.6\%) patients and (5.9\%) patients had both hypertension and diabetes mellitus. For $84(55.6 \%)$ of patients carotid Doppler ultrasound was done and atherosclerotic changes were reported in $46(54.7 \%)$ patients (Table 2$)$.

\section{Clinical presentations of stroke}

Regarding to clinical presentation, more than half (58.3\%) patients admitted to hospitals within 24 hours onset of stroke. The commonest complaint was failure to use hemi-body (80.7\%) followed by altered mentation (45.7\%). Twenty-eight(18.5\%) of the patients were having admission fever greater than $38^{\circ} \mathrm{C}$ and $\mathrm{RBS} \geq 200 \mathrm{mg} / \mathrm{dl}$ was recorded in $11(8 \%)$ of patients at admission (Table 3 ).

\section{Stroke sub types}

Ischemic (60.3\%) and hemorrhagic (39.7\%) were the most common stroke subtypes. The median age for the onset of ischemic and hemorrhagic stroke was 61.5 (IQR: 55-70) and 60(IQR: 60-75) years respectively. There was no statistically significant difference between two stroke subtypes with sex, area of residence, and preceding history of hypertension. Regarding to ischemic stroke cases the majority (76.6\%) had anterior vessel circulation affected territory followed by more than two areas (12\%) and Lacunars (7.8\%). In relation to anatomical location of hemorrhagic stroke, Basal ganglia (52.2\%), Lobar (22.6\%), and thalamic (19.3\%) were the most common findings.

\section{Complications}

Sixty four (42.8\%) patients had at least one complications. Increased intracranial pressure (ICP) (33.1\%) and aspiration (29.8\%) were the most common detected complications. About $10(6.6 \%)$ patients developed hospital acquired pneumonia and one patient was diagnosed with deep venous thrombosis (DVT). 


\section{In-hospital outcome at the stroke care unit}

The overall in-hospital mortality was 14 (9.3\%) with (95\% Cl: 5.2\%-15.1\%), and specific mortality to stroke subtypes of hemorrhagic (11.6\%) and Ischemic (7.7\%). Regarding to the cause of death, increased ICP $(50 \%)$, sepsis $(28 \%)$, and others were the commonly identified. The median length of hospital stay was 10 (IQR: 7-14) days.

Patients discharged to stroke care unit the degree of disability estimated based on several factors mentioned in the patients' charts. Based Modified Rankin Score scale, patient who had (mRS $\geq 3)$ at discharge were classified as poor post-stroke disability (greater functional impairment), from this study $55.6 \%$ with (95\% Cl: $47.3 \% 63.7 \%$ ) had poor disability outcome at discharge. Greater functional impairment were higher among hemorrhagic (61.6\%) compared to ischemic stroke (51.6\%).

\section{Factors associated with in-hospital outcome of stroke}

Finding from multivariable logistic regression analysis; sex and length of hospital stay were significant predictors of in-hospital mortality. Male stroke patients were $81 \%$ less likely to had in-hospital mortality compared to female patients ( $A O R=0.19,95 \% \mathrm{Cl}: 0.0380 .970$ ). Similarly stroke patients who had longer than 10 days in-hospital stay the odds of mortality decreased by $79 \%$ (AOR $=0.21,95 \% \mathrm{Cl}: 0.0480 .93$ ) compared to those who had LOS $\leq 10$ dyas.

Whereas evidence of increased intracranial pressure was predictor of poor post-stroke disability outcome at multivariable logistic regression analysis model. Accordingly patients with evidence of increased intracranial pressure the odds of poor post-stroke disability 3.18 (AOR=3.18, 95\% Cl: 1.437 .05$)$ times higher compared to those not have ICP (Table 4).

\section{Discussion}

The most common stroke subtype was ischemic stroke (60.3\%) cases while the remaining accounted for hemorrhagic stroke. The median age of patients with ischemic stroke was 65.5 years (IQR: 60.5-78) and compared to 59.4 years (IQR: 55-70) for hemorrhagic stroke. Compared to previous study that was done in the same setup and study done in southern Ethiopia the proportion of hemorrhagic stroke was found to be higher in our study $[15,16]$. But the proportion is comparable with other studies done in SSA[17, 18]. Hypertension was found in half of our patients with similar proportion between stroke subtypes. Atrial fibrillation was also found in 29(37.6\%) of ischemic stroke patients who had Electrocardiogram (ECG) reading. Cardiac abnormalities were found in $63(80.7 \%)$ of ischemic stroke patients who had Echocardiography, Degenerative Valvular disease (DVHD) being the commonest. Only 3 patients had chronic rheumatic valvular heart disease (CRVHD) and all of them were having ischemic stroke.

The overall in-hospital stroke mortality in this study was $9.3 \%$. The mortality of ischemic stroke $(7.7 \%)$ whereas that of hemorrhagic stroke was (11.7\%). The overall in-hospital stroke mortality in this study was 
higher than that of high income countries such as Germany (5.4\%)[14], United States of America (6\%)[19], and China (2.30\%)[20]. This may be explained by difference in health seeking behavior, professional expertise, severity of the disease, and stoke care unit setup.

Mortality from this study was lower than reports from in-hospital mortality from general wards at St. Paul's teaching hospital (30.1\%)[21], and Lusaka, Zambia (40.4\%)[18], Ghana (41.1\%)[17, 22], Nigerian teaching hospital (35\%)[23], and South Africa (33\%)[13]. This might be due to the difference in socio demographic characteristics and set up difference. In all the above mentioned settings stroke care were given in general medical wards where there is no dedicated staffs and materials for appropriate interventions of stroke. In addition this finding may underscore the advantage of establishing stroke care unit. Moreover, stroke mortality of this study was lower than a previous reports of stroke care units of South Africa (16\%)[13] and Denmark (21\%)[24]. This could be explained by difference in severity of the disease, professional expertise and year of the study.

Compared to the previous study done among 98 stroke patients in similar setup before establishment the unit, mortality and median length of hospital stay seems lower (9.3 vs. $13.3 \%$ ) and (10 vs.13 days) respectively[16]. Regarding to the characteristics of patients before and after establishment of stroke care unit were more or less similar except high proportion of ischemic stroke patients from previous study $(69.3 \%)$ compared to ours $(60.3 \%)$ This might be explained by management of cases of stroke through organized stroke care unit and sample size difference.

This study also identified the commonest causes of death were increased ICP (50\%) and sepsis (26.6\%). Half of the total deaths occurred in the first seven days, increased ICP being the commonest cause. Sepsis and increased ICP contributed equally for deaths occurred after seven days of hospital stay. Regarding post stroke disability, about half (55.6\%) patients were discharged with severe disability with greater functional impairment (Poor disability MRS score $\geq 3$ ). This finding was consistent with study conducted Tanzania (53\%)[25] and China (53\%)[26]. This finding was also higher than study conducted in Germany[14]. In fact this difference might be explained through study population and health care system difference between Ethiopia and Germany. In addition limited rehabilitation services in our hospital which is the case for most low-income countries. More over in our setup stroke patients are regularly followed by nurses and neurologists but physical therapists rarely.

The main predictors of in-hospital stroke mortality in this study; male sex, and length of hospital stay. Male patients were less likely to die compared to female patients. The possible explanations might be due age difference and atypical presentations between male and female patients. This finding was supported by other studies[24]. Patients who stayed admitted longer than 10 days stroke mortality was decreased by $79 \%$ compared to those who stay admitted less than 10 days. This may be explained by early death from acute stroke complication such as Increased ICP and most patients were from rural areas which might be responsible to death on hospital arrival.

The main predictors of poor outcome was evidence of increased ICP. Patients who have increased ICP were three times more likely to have poor in-hospital outcome as compared to those without features of 
increased ICP.

Firstly, this study was limited by inadequate sample size which might affect the power of study. Secondly, this study did not show the actual overall stroke mortality of the hospital, which excluded deaths reported out of stroke care unit like deaths at emergency departments on arrival. In addition, our stroke care unit is not separated. In addition, patient discharge against medical advice was also a key barrier affecting optimal clinical care. Key skills for stroke care, such as speech and language therapy for dysphagia assessment, are not available. Limited staff especially stroke specialists (e.g., neurologists, neurosurgeons and trained stroke nurses) can also contribute.

\section{Conclusion}

Ischemic stroke was the most common stroke subtype. In-hospital mortality was relatively lower. However, greater functional impairment was high at discharge. Stroke morality was observed lower among male and longer in-hospital stay patients, while evidence of increased intracranial pressure was associated with poor post-stroke disability at discharge.

\section{Declarations}

\section{Ethical approval and consent to participate}

The study was based on secondary data from medical records of stroke patients. Ethical clearance was obtained from institutional ethical review board of school of medicine, college of medicine and health science, University of Gondar. Waiver letter obtained from University of Gondar comprehensive specialized hospital administration. Checklists were locked in cabinets, databases and the database was password protected.

\section{Consent for publication}

Not applicable

\section{Availability of data and materials}

The data are available and could be accessed from the corresponding author when asked with reasonable inquiry.

\section{Competing interests}

The authors declare that they have no competing interests 


\section{Funding}

The study was funded by University of Gondar, Ethiopia. The funder has no role in study design, data collection and analysis, interpretation of data, decision to publish, or preparation of the manuscript.

\section{Authors' contributions}

SGA, NBG, ESG, and KST participated to design the study, data collection, performed data analysis, visualization, validation the whole work and prepared the manuscript. SGA took part in funding acquisition, data collection, supervision and software and other resources. All authors read and approved the final manuscript.

\section{Acknowledgement}

We would like to acknowledge all participants, data collectors and hospital administrator.

\section{Abbreviations}

CRVHD: Chronic Rheumatic Valvular Heart Disease, DALY: Disability Adjusted Life's of Year, DVHD: Degenerative Valvular Heart Disease, VT: Deep Venous Thrombosis, ECG: Electro Cardiogram, ICH: Intra Cranial Hemorrhage, ICP: Intra Cranial Pressure, IQR: Inter Quartile Range, IRB: Institutional Review Board, LMIC: Low and Middle Income Country, LOS: Length of Hospital Stay, MRS: Modified Rankin Score, NCD: Non Communicable Disease, OR: Odds Ratio, TIA: Transient Ischemic Attack.

\section{References}

1. Yusuf, S., et al., Global burden of cardiovascular diseases: Part Il: variations in cardiovascular disease by specific ethnic groups and geographic regions and prevention strategies. Circulation, 2001. 104(23): p. 2855-2864.

2. Mensah, G.A., Epidemiology of stroke and high blood pressure in Africa. Heart, 2008. 94(6): p. 697-705.

3. Thrift, A.G., et al., Global stroke statistics. International Journal of Stroke, 2014. 9(1): p. 6-18.

4. Malani, P.N., Harrison's principles of internal medicine. JAMA, 2012. 308(17): p. 1813-1814.

5. Norrving, B. and B. Kissela, The global burden of stroke and need for a continuum of care. Neurology, 2013. 80(3 Supplement 2): p. S5-S12.

6. Feigin, V.L., et al., Global burden of stroke and risk factors in 188 countries, during 1990-2013: a systematic analysis for the Global Burden of Disease Study 2013. The Lancet Neurology, 2016. 15(9): p. 913-924. 
7. Jowi, J. and P. Mativo, Pathological sub-types, risk factors and outcome of stroke at the Nairobi Hospital, Kenya. East African medical journal, 2008. 85(12).

8. Govan, L., P. Langhorne, and C.J. Weir, Does the prevention of complications explain the survival benefit of organized inpatient (stroke unit) care? Stroke, 2007. 38(9): p. 2536-2540.

9. Indredavik, B., et al., Treatment in a combined acute and rehabilitation stroke unit. Stroke, 1999. 30(5):

p. 917-923.

10. Liu, L., et al., Stroke and stroke care in China. Stroke, 2011. 42(12): p. 3651-3654.

11. Tamm, A., et al., Impact of stroke care unit on patient outcomes in a community hospital. Stroke, 2014. 45(1): p. 211-216.

12. Langhorne, P., L. de Villiers, and J.D. Pandian, Applicability of stroke-unit care to low-income and middle-income countries. The Lancet Neurology, 2012. 11(4): p. 341-348.

13. Villiers, L.d., S.Z. Kalula, and V.C. Burch, Does multidisciplinary stroke care improve outcome in a secondary-level hospital in South Africa? International Journal of Stroke, 2009. 4(2): p. 89-93.

14. Koennecke, H.-C., et al., Factors influencing in-hospital mortality and morbidity in patients treated on a stroke unit. Neurology, 2011. 77(10): p. 965-972.

15. Deresse, B. and D. Shaweno, Epidemiology and in-hospital outcome of stroke in South Ethiopia. Journal of the neurological sciences, 2015. 355(1): p. 138-142.

16. Greffie, E.S., T. Mitiku, and S. Getahun, Risk factors, clinical pattern and outcome of stroke in a referral hospital, Northwest Ethiopia. Clinical Medicine Research, 2015. 4: p. 6,182-188.

17. Agyemang, C., et al., Stroke in Ashanti region of Ghana. Ghana medical journal, 2012. 46(2): p. 12-17.

18. Atadzhanov, M., et al., Stroke characteristics and outcomes of adult patients admitted to the University Teaching Hospital, Lusaka, Zambia. The Open General and Internal Medicine Journal, 2012. $5(1)$.

19. Bravata, D.M., et al., Processes of care associated with acute stroke outcomes. Archives of Internal Medicine, 2010. 170(9): p. 804-810.

20. He, Q., et al., Trends in in-hospital mortality among patients with stroke in China. PloS one, 2014. 9(3): p. e92763.

21. Gedefa, B., et al., Assessment of Risk Factors and Treatment Outcome of Stroke Admissions at St. Paul's Teaching Hospital, Addis Ababa, Ethiopia. J Neurol Neurophysiol, 2017. 8(431): p. 2. 
22. Sarfo, F.S., et al., Trends in stroke admission and mortality rates from 1983 to 2013 in central Ghana. Journal of the neurological sciences, 2015. 357(1-2): p. 240-245.

23. Ekeh, B., et al., Stroke mortality and its predictors in a Nigerian teaching hospital. African health sciences, 2015. 15(1): p. 74-80.

24. Svendsen, M.L. and S.P. Johnsen, In-Hospital Medical Complications, Length of Stay, and Mortality Among Stroke Unit Patients. 2011.

25. Wibecan, L., et al., Post-Stroke Disability at a National Referral Hospital in Tanzania (P3.322). Neurology, 2017. 88(16 Supplement): p. P3.322.

26. Yang, Y., et al., The disability rate of 5-year post-stroke and its correlation factors: a national survey in China. PLoS One, 2016. 11(11): p. e0165341.

\section{Tables}

Table 1: Socio demographic characteristics of stroke patients admitted to stroke care unit at university of Gondar comprehensive specialized hospital,2017 ( $n=151)$

\begin{tabular}{lll} 
Characteristics & Frequency & Percentage \\
\hline Gender & & \\
\hline Male & 75 & 49.7 \\
\hline Female & 76 & 50.3 \\
\hline Age & & \\
\hline $20-39$ & 10 & 6.6 \\
\hline $40-64$ & 59 & 39.1 \\
\hline$\geq 65$ & 82 & 54.3 \\
\hline Religion & & \\
\hline Muslim & 17 & 11.3 \\
\hline Orthodox Christian & 134 & 88.7 \\
\hline Residence & & \\
\hline Urban & 53 & 35.1 \\
\hline Rural & 98 & 64.9
\end{tabular}

Table 2: Socio-demographic characteristics of stroke patients admitted at university of Gondar comprehensive specialized hospital stroke care unit, $2017(n=151)$ 


\begin{tabular}{lll} 
Risk factors & Frequency & Percentage (\%) \\
\hline Hypertension & 76 & 50.3 \\
\hline Diabetes mellites & 13 & 8.6 \\
\hline Previous stroke or TIA & 23 & 15.2 \\
\hline Structural heart disease & 74 & 49 \\
\hline Smoking & 2 & 1.3 \\
\hline More than moderate alcohol & 15 & 10.6 \\
\hline HIV positive & 4 & 2.6 \\
\hline Carotid atherosclerotic change & 46 & 54.7 \\
\hline Atrial fibrillation & 33 & 28.7
\end{tabular}

Table 3: Clinical presentations of stroke patients admitted at university of Gondar comprehensive specialized hospital stroke care unit, $2017(n=151)$.

\begin{tabular}{lll} 
Characteristics & Frequency & Percentage (\%) \\
\hline Hemi paresis/hemiplegic & 112 & 80.8 \\
\hline Failure to communicate & 57 & 37.7 \\
\hline Dysphasia & 30 & 19.9 \\
\hline Incontinence & 65 & 43 \\
\hline Failed admission swallow test & 76 & 50.3 \\
\hline Headache & 58 & 38.4 \\
\hline Fever $\geq 38$ & 28 & 18.5 \\
\hline Vomiting & 40 & 26.5 \\
\hline Seizure & 15 & 10 \\
\hline Altered mentions & 69 & 45.7
\end{tabular}

Table 4: Multivariable logistic regression analysis of predictors of in hospital outcome of stroke patients admitted at stroke care unit of Gondar university comprehensive specialized hospital. 
Characteristics

In hospital

mortality

Yes
Adjusted OR

No
Poor outcome

Yes

Aspiration

pneumonia

$\begin{array}{lllllll}\text { Yes } & 8 & 37 & \begin{array}{l}2.14(0.45 \\ 10.21)\end{array} & 32 & 13 & \begin{array}{l}1.12(0.45 \\ 2.76)\end{array} \\ \text { No } & 6 & 100 & 1 & 52 & 54 & 1\end{array}$

Admission swallow

test

Passed

$2 \quad 75$

$0.12(0.01$

32

$45 \quad 0.49(0.21$

1.01)

1.11)

Failed

12

$\begin{array}{ll}62 & 1\end{array}$

52

221

Any incontinence

Yes

12

53

$5.57(0.98$

31.42)

45

20

$1.67(0.76$

3.68)

No

2

$84 \quad 1$

39

$47 \quad 1$

Mentation

Intact

3

79

$0.95(0.14$

6.69)

37

$45 \quad 0.64(0.28$

1.46)

Altered

11

$58 \quad 1$

47

221

Increased ICP

\begin{tabular}{|c|c|c|c|c|c|c|}
\hline Yes & 7 & 43 & $\begin{array}{l}2.53(0.49 \\
13.0)\end{array}$ & 37 & 13 & $\begin{array}{l}3.18(1.43 \\
7.05)^{\star}\end{array}$ \\
\hline No & 7 & 94 & 1 & 47 & 54 & 1 \\
\hline \multicolumn{7}{|l|}{ Sex } \\
\hline Male & 3 & 72 & $\begin{array}{l}0.19(0.038 \\
0.97)^{\star}\end{array}$ & 40 & 35 & \\
\hline Female & 11 & 65 & 1 & 44 & 32 & $\begin{array}{l}0.81(0.39 \\
1.68)\end{array}$ \\
\hline
\end{tabular}

Length of hospital stay

\begin{tabular}{lllllll}
$\leq 10$ days & 11 & 75 & 1 & 42 & 44 & 1 \\
\hline$>10$ days & 3 & 62 & $\begin{array}{l}0.21(0.048 \\
0.93)^{*}\end{array}$ & 42 & 23 & $\begin{array}{l}1.62(0.78 \\
3.36)\end{array}$
\end{tabular}


* shows statistically significant association at a p-value $<0.05$ 